\title{
SIT: Bessere Compliance dank nur vier Injektionen
}

_ In der Behandlung der Allergischen Rhinitis spielt die Auswahl der geeigneten spezifischen Immuntherapie (SIT) eine entscheidende Rolle. Denn die jeweiligen Eigenschaften des verordneten Medikaments beeinflussen neben Wirksamkeit und Verträglichkeit auch unmittelbar die Compliance.

Vorteile gegenüber einer Langzeit-Immuntherapie bietet hier das Präparat POLLINEX ${ }^{\circledR}$ Quattro, mit dem lediglich vier Sitzungen

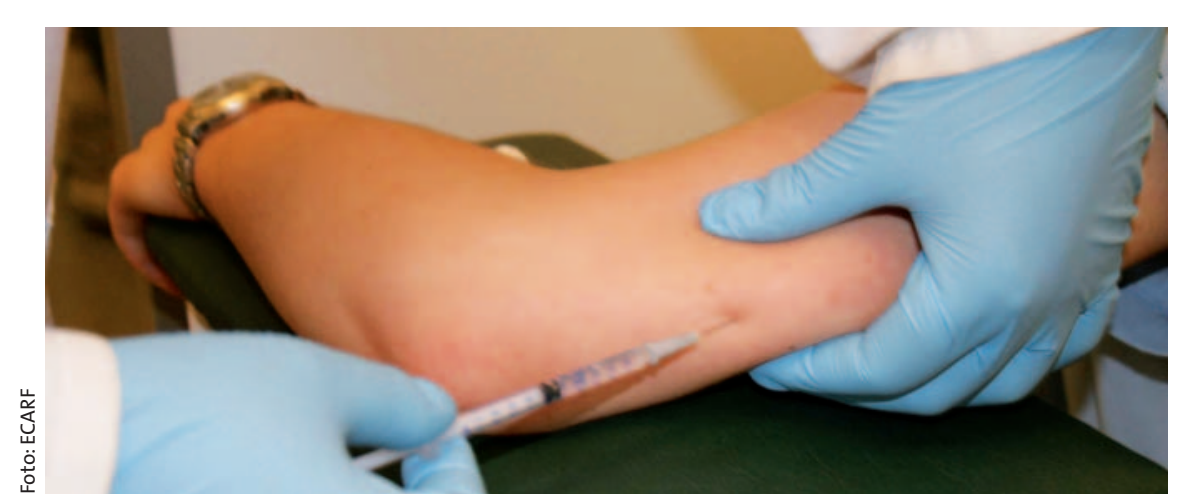

Je weniger Spritzen bei einer spezifischen Immuntherapie, desto höher die Compliance.

erforderlich sind. Die kurze Therapiedauer wird durch den dualen Wirkansatz von Allergoid und dem Adjuvans $M P L^{\circledR}$ ermöglicht, wodurch die TH-1-Immunantwort verstärkt wird. Dr. Jens Vollmar, München, stellte Daten zu Wirkweise und Anwendungsgebieten des Lipopolysaccharid-Derivats vor. So ist MPL ${ }^{\circledR}$ unter anderem Bestandteil des HPVImpfstoffs von GlaxoSmithKline.

Dr. Dietrich Stollewerk, Köln, präsentierte Daten eines Vergleichs zweier Therapieregimes.
233 Baum- und Pollenallergiker zwischen 9 und 75 Jahren wurden entweder mit einer Langzeit-SIT oder einer Komplett-Therapie mit vier Injektionen (POLLINEX ${ }^{\circledR}$ Quattro) behandelt. Die Studienteilnehmer durften in den drei vorangegangenen Jahren keine SIT erhalten haben. Ziel der deskriptiven Studie war, die Äquivalenz beider Methoden nachzuweisen. Die Ergebnisse zeigten, dass die Kurzzeit-SIT der Langzeit-SIT sowohl bei der Augen- als auch bei der Nasensymptomatik nicht unterlegen war. Nebenwirkungen beschränkten sich bei der Kurzzeittherapie auf lokale Events. Stollewerk hält daher die komplette Immuntherapie mit lediglich vier Injektionen bezüglich Compliance und Kosten einer Langzeittherapie für überlegen.

slx

Satellitensymposium „Komplett und flexibel: Die Immuntherapie mit 4 Injektionen. Wieviel SIT kommt beim Patienten an?" im Rahmen des 4. Gemeinsamen Deutschen Allergiekongresses, Berlin, 5. September 2009

Veranstalter: Bencard Allergie, München

\section{Metastasierte und rezidivierte Kopf-Hals-Tumoren: neuer Standard definiert}

_ Über $60 \%$ aller Patienten mit Kopf-HalsTumoren haben bereits bei Erstdiagnose eine lokal fortgeschrittene, inoperable Erkrankung. Nach lokoregionaler Therapie entwickeln bis zu 80\% Lokalrezidive oder Fernmetastasen. Auch mit Polychemotherapien liegt das mediane Überleben in diesem großen Kollektiv nur bei 6-8,5 Monaten, berichtete Dr. Dirk Arnold, Halle. Beim Therapieentscheid standen bislang Symptomkontrolle und Nebenwirkungsprofil im Fokus.

Die randomisierte EXTREME-Studie ist hier ein großer Schritt nach vorn. Seit 30 Jahren wurde erstmals wieder ein Überlebensvorteil bei rezidivierten und metastasierten Kopf-Hals-Tumoren erreicht. Die internationale Phase-III-Studie schloss rund 450 Patienten ein, die randomisiert entweder die bisherige Standardtherapie mit Cisoder Carboplatin und 5-Fluorouracil in dreiwöchigen Zyklen oder die gleiche Chemotherapie plus Cetuximab (Erbitux ${ }^{\circledR}$ ) erhielten. Im Cetuximab-Arm wurde der Antikörper nach maximal sechs Zyklen als Erhaltungstherapie weiter verabreicht. In der ersten Studienphase konnten die Patienten im Schnitt 18 Wochen mit Cetuximab behandelt werden; dabei erhielten $84 \%$ der Patienten mindestens $80 \%$ der geplanten Dosis. Die anschließende Erhaltungstherapie war bei 100 Patienten (45\%) machbar, berichtete Arnold.

Die zusätzliche Antikörpergabe verlängerte das Gesamt-Überleben um fast drei Monate (10,1 Monate im Cetuximab-Arm versus 7,4 Monate im Kontrollarm). Das progressionsfreie Überleben verbesserte sich von 3,3 auf 5,6 Monate. Subgruppenanalysen zeigen, dass praktisch alle Patienten von Cetuximab zusätzlich zur Chemotherapie profitierten. Grundsätzlich, so Arnold, scheint die Cisplatin-haltige Therapie in Bezug auf das Ansprechen etwas effektiver zu sein als das Carboplatin-Regime.

Relevante Nebenwirkungen vom Grad 3/4 und die Lebensqualität waren in beiden Armen vergleichbar. Erwartungsgemäß waren unter Cetuximab Hautreaktionen signifikant häufiger als bei Kontrollpatienten. Anders als beim Bronchialkarzinom war der Rash aber kein Effektivitätsprädiktor. Auch gab es keine Korrelation zwischen EGFRExpression und Gesamt-Überleben. arn

Satellitensymposium „Neue Konzepte in der Erstlinienbehandlung von kolorektalen/ Bronchial- und Kopf-Hals-Tumoren "im Rahmen der DGHO-Jahrestagung 2009 Mannheim, 2. Oktober 2009

Veranstalter: Merck Serono, Darmstadt 